OPEN ACCESS

Edited by:

Jorge Matias-Guiu,

Complutense University of

Madrid, Spain

Reviewed by:

Rishein Gupta,

University of Texas at San Antonio,

United States

Xing-huai Sun,

Eye \& ENT Hospital,

Fudan University, China

*Correspondence:

Wenyi Guo

wyguo@163.com

$\mathrm{LiXu}$

x_jenny_dr_2014@sina.com

†These authors have contributed equally to this work

Specialty section: This article was submitted to

Multiple Sclerosis and

Neuroimmunology,

a section of the journal

Frontiers in Immunology

Received: 03 July 2019 Accepted: 03 January 2020 Published: 28 January 2020

Citation:

Peng $C$, Wu Y, Ding $X$, Chen $D$,

Zeng C, Xu L and Guo W (2020)

Characteristic Cytokine Profiles of

Aqueous Humor in Glaucoma

Secondary to Sturge-Weber Syndrome. Front. Immunol. 11:4. doi: 10.3389/fimmu.2020.00004

\section{Characteristic Cytokine Profiles of Aqueous Humor in Glaucoma Secondary to Sturge-Weber Syndrome}

\author{
Cheng Peng ${ }^{1,2+}$, Yue $\mathrm{Wu}^{1,2+}$, Xuming Ding ${ }^{1}$, Di Chen ${ }^{1,2}$, Changjuan Zeng ${ }^{1}, \mathrm{Li} \mathrm{Xu}^{1 *}$ and \\ Wenyi Guo ${ }^{1,2 *}$
}

'Department of Ophthalmology, Ninth People's Hospital, Shanghai Jiao Tong University School of Medicine, Shanghai, China, ${ }^{2}$ Shanghai Key Laboratory of Orbital Diseases and Ocular Oncology, Shanghai, China

Patients with Sturge-Weber syndrome (SWS) are susceptible to ocular complications, and among them, glaucoma is one of the most frequent forms. In current study, we utilized multiplex human cytokine antibody array to simultaneously measure the concentration of 40 cytokines in aqueous humor $(\mathrm{AH})$ of patients with SWS-induced glaucoma (SG), or from patients with senile cataract as controls. Compared with the control group, levels of interleukin (IL)-12p40, macrophage inflammatory protein (MIP)-1d, tumor necrosis factor-alpha (TNF-a), IL-5, IL-7, interleukin-6 receptor (IL-6R), and $B$ lymphocyte chemoattractant $(B L C)$ in $A H$ were significantly higher in SG group. Samples from SG patients displayed significantly lower levels of MIP-1b, IL-6, MIP-1a, and monocyte chemoattractant protein (MCP)-1 than controls. Further analysis showed that IL-7, MIP-1a, TNF-a were positively correlated with intraocular pressure (IOP) in patients with early-onset SG. Moreover, IL-12p40 was negatively correlated with age in patients with SG. These cytokines may make contributions to the immunopathogenesis or progression of glaucoma in patients with SWS.

Keywords: sturge-weber syndrome, secondary glaucoma, cytokines, intraocular pressure, aqueous humor

\section{INTRODUCTION}

Sturge-Weber syndrome is a rare neurocutaneous disorder, which can be present sporadically and is characterized by vascular malformation usually composed of a unilateral facial port-wine stain (PWS) with ipsilateral deformed leptomeningeal vasculature as well as a choroidal hemangioma (1). Previous study showed that SWS is caused by the somatic mosaic mutation in GNAQ (2). Moreover, the severity of vascular malformation in SWS possibly depends on the timing of GNAQ mutation in the course of embryofetal development (3).

Inflammatory factors have been widely implicated in pathological process of many neurological disorders $(4,5)$. As for neurocutaneous syndrome, Neurofibromatosis-1(NF1), tuberous sclerosis complex (TSC) as well as SWS are the most common types, (6) and altered cytokine level has been previously reported in specimen of NF1 and TSC. Compared with healthy controls, the level of the inflammatory cytokines (IFN- $\gamma$, TNF- $\alpha$, IL-6) in serum of NF1 patients are significantly higher, indicating the great importance of hyperinflammation in pathogenesis of NF1 (7). Similarly, significant proinflammatory cytokine expression in tuber of tuberous sclerosis complex (TSC) patients has been shown in either protein (8) or gene (9) level. Therefore, we assume that altered local cytokine profile can also be detected in SWS patients, which may contribute to pathogenesis and progression of the disease. 
Secondary glaucoma is the major type of ocular complications in SWS, and its prevalence ranges from 30 to $70 \%$ in SWS patients (10). SG patients demonstrate a bimodal distribution. According to the onset age for glaucoma, $60 \%$ of them demonstrate an early onset in infancy and early childhood, whereas $40 \%$ of them demonstrate a late onset in late childhood and early adulthood (11). Pathogenesis of glaucoma in patients with SWS has been previously reported to be mainly associated with angle dysgenesis (10), elevation of episcleral venous pressure (EVP) (12), as well as an alteration in ophthalmic hemodynamics (13). Additionally, it is assumed that different pathogenesis may contribute to the bimodal-onset glaucoma. When it comes to early-onset glaucoma, angle dysgenesis plays a crucial role. On the other hand, in the late-onset form, elevated EVP that possibly results from dilated episcleral veins, is the main pathogenic factor (14). Nevertheless, the exact mechanism of elevated intraocular pressure (IOP) in SWS-induced glaucoma (SG) still remains unclear.

For glaucoma, morphological and functional changes in the trabecular meshwork (TM) are believed to be mainly responsible for increased resistance of AH outflow, and consequently lead to elevation of IOP $(15,16)$. Predominantly, these changes in the TM include the alteration in amount and the quality of the extracellular matrix (ECM) (17). Also, alteration in infiltrated immune cells, like neutrophils and macrophages, have been detected in the TM of various glaucomatous eyes $(18,19)$. In addition, it has been reported previously that endothelial cells of TM can secrete various factors and cytokines which play a pivotal role in regulation and modulation of ECM remodeling, and therefore affect the pathway of AH outflow (20,21). Most recently, specific HSP-T cell response has been reported to play a pivotal role in IOP-independent glaucoma progression (22). Therefore, we hypothesize that immune factors may be the cause rather than the result of glaucoma in SWS patients.

To summarize, we speculated that changes in the cytokine-mediated signaling pathway can reflect the extent of glaucomatous development in SWS patients. Hence, detecting cytokine concentrations in the AH of SG patients may provide more insight into the local pathogenetic mechanism of this unique glaucoma subtype. In the present study, we utilized a Quantibody Human Inflammation Array 3 (Ray Biotech, Inc, Norcross, GA, USA) to investigate the cytokine profile in $\mathrm{AH}$ of SG patients.

\section{MATERIALS AND METHODS}

\section{Ethics Statement}

This study was approved by the Institutional Review Board of Ninth People's Hospital, Shanghai Jiao Tong University School of Medicine, which followed the tenets of the Declaration of Helsinki. All methods were performed according to relevant guidelines and protocols, including any relevant details. Written informed consent was obtained for each participant. In case of underaged SG patients, written informed consent was obtained from parents or legal guardian.

\section{Subjects}

In this cross-sectional study, diagnosis of SWS-induced glaucoma (SG) was based on general anesthesia examination for infant patients. IOP was not implemented as the decisive factor for final diagnosis, since the newborns presented lower level of IOP compared to adults. Close observation was given when an IOP above $21 \mathrm{~mm} \mathrm{Hg}$ or apparent asymmetry between two eyes (above $6 \mathrm{mmHg}$ ) occurred. A cup-to-disc ratio (C/D) above 0.5, apparent asymmetry C/D between two eyes, corneal enlargement, and other corneal abnormalities, like Haab striae or corneal edema are strong diagnostic indicators of glaucoma (23). Patients undergoing routine surgery for uncomplicated, senile cataract were enrolled as controls.

For SWS patients, the clinical parameters (IOP, C/D, corneal diameters) were collected under general anesthesia before the glaucoma surgery. Agents used in the procedure of general anesthesia were stated below: sevoflurane or propofol for induction of anesthesia and either sevoflurane plus remifentanil or propofol plus remifentanil for maintenance of anesthesia. Measurement of IOP was carried out with an AccuPen Applanation Tonometer (Accutome, Inc, Malvern, Pennsylvania, USA). For all patients, a history of ocular or systemic inflammation stood for an exclusion criterion. For infant subjects, other systemic vascular malformations were ruled out except for PWS, and all babies followed the same routine vaccine scheme of China ensuring a similar vaccine-induced immunological background. Moreover, for enrolled individual patients, only one eye was included. The diagnosis of glaucoma, surgery and collection of $\mathrm{AH}$ sample and clinical data were all performed by the same doctor (W.Y. G.).

\section{Collection of Aqueous Humor}

For both SG patients and controls, AH samples (50-100 $\mu \mathrm{L})$ were collected at the start of surgery, by carrying out paracentesis with a $1.0 \mathrm{~mL}$ syringe and attached 30 -gauge needle. After collection, all samples were instantly transferred and stored at $-80^{\circ} \mathrm{C}$ for further cytokine analysis.

\section{Cytokine Antibody Array}

The concentrations of 40 selected inflammatory cytokines was measured using a multiplex quantitative cytokines array (Ray Biotech, Inc, Norcross, GA, USA). The cytokines array was performed according to the manufacturer's guidelines. Samples were detected through use of a GenePix scanner (Axon Instruments, Inc, Foster City, CA, USA), and further analyzed with a GenePix Pro 6.0 software (Axon Instruments, Inc).

\section{Statistical Analysis}

Principal component analysis (PCA) was carried out on cytokine concentrations after log transformation. Cluster analysis was carried out through use of 1-Pearson's correlation as well as Ward's hierarchical clustering. Pathway analysis was performed to investigate the significant pathways enriched by the differentially expressed cytokines according to Kyoto Encyclopedia of Genes and Genomes (KEGG) (http://www. genome.jp/kegg/). The PCA, cluster analysis and KEGG pathway analysis were performed through use of the $\mathrm{R}$ statistical package 
(http://www.r-project.org). Additional statistical analysis was performed using Prism for Windows software (Version 7.00, Graphpad Software, Inc.) and SPSS (Version 25.00, IBM SPSS Statistics, Inc.). The Shapiro-Wilk test was used to assess whether the data showed a normal distribution. Spearman's correlation analyses were used to evaluate the correlations between different cytokine levels, as well as correlations between concentrations and age, IOP, C/D and corneal diameter. MannWhitney $\mathrm{U}$ tests were used to evaluate the difference in cytokine levels between the groups (SG and control). Multiple linear regression was performed in subgroup analysis for adjustment of demographic differences.

Data were expressed as means and standard deviation (SD) or medians and interquartile range (IQR). A $P<$ 0.05 was considered to be statistically significant, except for distinction of differentially expressed cytokine, for which a Bonferroni correction was applied. Because there were 40 different comparisons, a P-value less than 0.00125 (i.e., $P$ $=0.05 / 40)$ was considered to be statistically significant with Bonferroni correction.

\section{RESULTS}

\section{Demographic Data}

Overall, 22 SWS-induced glaucoma (SG) patients and 15 subjects with senile cataract were included in the present study. The demographic data including number of patients, age, sex, IOP, $\mathrm{C} / \mathrm{D}$, and corneal diameter, are summed up in Table 1 . The median age of the SWS group and the control group were 2 years old and 77 years old, respectively $(P<0.001)$. No significant difference was observed in the sex distribution between the SG and the control groups $(P=0.7384)$.

\section{Cluster Analysis and Principal Component Analysis}

Cluster analysis was utilized to obtain the overview of cytokine profile in the $\mathrm{AH}$ samples. The result showed that

TABLE 1 | Demographic data

\begin{tabular}{|c|c|c|c|}
\hline & SG & Control & $P$-value \\
\hline Number of eyes & 22 & 15 & \\
\hline $\begin{array}{l}\text { Age [years; } \\
\text { median (IQR)] }\end{array}$ & $2(0.52,3.75)$ & $77(71,83.5)$ & $<0.0001^{*}$ \\
\hline Sex (Female: Male) & $11: 11$ & $9: 6$ & $0.7384^{+}$ \\
\hline $\begin{array}{l}\mathrm{IOP}(\mathrm{mm} \mathrm{Hg} \\
\text { mean } \pm \mathrm{SD})\end{array}$ & $20.41 \pm 9.4$ & $14.53 \pm 3.38$ & $0.0119^{\#}$ \\
\hline C/D [median (IQR)] & $0.725(0.6125,0.8)$ & n. a. & \\
\hline $\begin{array}{l}\text { Cornea diameter } \\
\text { (mm; mean } \pm \text { SD) }\end{array}$ & $12.64 \pm 0.92$ & n. a. & \\
\hline
\end{tabular}

Control, senile cataract eyes; SG, Sturge-weber syndrome induced glaucoma; $I O P$, intraocular pressure; $C / D$, cup-to-disc ratio; $I Q R$, interquartile range, shown as (25\%, 75\%);

n. a., not applicable;

*Mann-Whitney U-test, \# Welch's t-test, + Fisher's Exact test.

$P<0.05$ was considered significant (bold and italic). all AH samples can be separated into two major groups, and the two clusters isolated basically through the different expression of cytokines (Figure 1A). In addition, this analysis was confirmed by using principal component analysis (PCA). PCA data showed that the SWS group had a distinct component of different cytokines when compared to the control group (Figure 1B).

\section{Differences in the Cytokine Profile in the Aqueous Humor}

Table 2 shows the concentrations of 40 cytokines. Eleven cytokines were significantly expressed differently between two groups (Figure 2). Compared with controls, the SG group had a notably increased level of IL-12p40 $(P<0.0001)$, MIP-1d $(P$ $<0.0001)$, IL-7 $(P<0.0001)$, IL-6R $(P<0.0001)$, BLC $(P<$ $0.0001)$, IL-5 $(P<0.0001)$, and TNF-a $(P<0.0001)$. Moreover, the cytokine concentration of MIP-1b $(P<0.0001)$, IL-6 $(P<$ $0.0001)$, MIP-1a $(P=0.0001)$, MCP-1 $(P=0.0011)$ were notably lower in the SG group.

Table 3 presents the correlation among the eleven most differently expressed cytokines. Positive correlation was found between most of the eleven differently expressed cytokines, and there was no negative correlation between any of these cytokines.

Table S2 demonstrates the result of subgroup analysis. In order to adjust the cytokine level with age, we used multiple linear regression in both SG group(infants) and cataract group(elders) separately. The data demonstrated that age did not influence the cytokine level except for IL12-p40 and IL-6R in the SG group, and the demographic data did not affect any cytokine level in the cataract group. Therefore, the result suggested that the difference in the cytokine expression is mainly due to the disease itself instead of age-caused bias.

\section{Correlation of Cytokine Levels With Clinical Parameters}

In order to explore the direct association between cytokine levels and clinical parameters, we performed spearman correlation test in the SG group. Except for the negative correlation between concentration of IL-12p40 and age of patients, there is no statistically significant correlation between cytokine level and clinical parameters, such as age, IOP, C/D and corneal diameter $(P=0.0046$; Table S1).

We further selected the patients who had surgery before the age of four as the candidates of early-onset SG group, and carried out the same correlation analysis (Table 4). Interestingly, we noticed that the concentration of three cytokines were remarkably correlated with IOP in the early-onset SG patients (IL-7, MIP-1a and TNF-a; $\rho=0.5824,0.5277$, $0.5455 ; P=0.0179,0.0356,0.0289$, respectively; Figure 3 ). Additionally, concentration of IL-12p40 was negatively correlated with age in early-onset SG patients $(\rho=-0.7114$; $P=0.0028$; Figure 3).

\section{KEGG Pathway Analysis}

We further performed KEGG pathway analysis to investigate the significant pathways enriched by the differentially expressed 


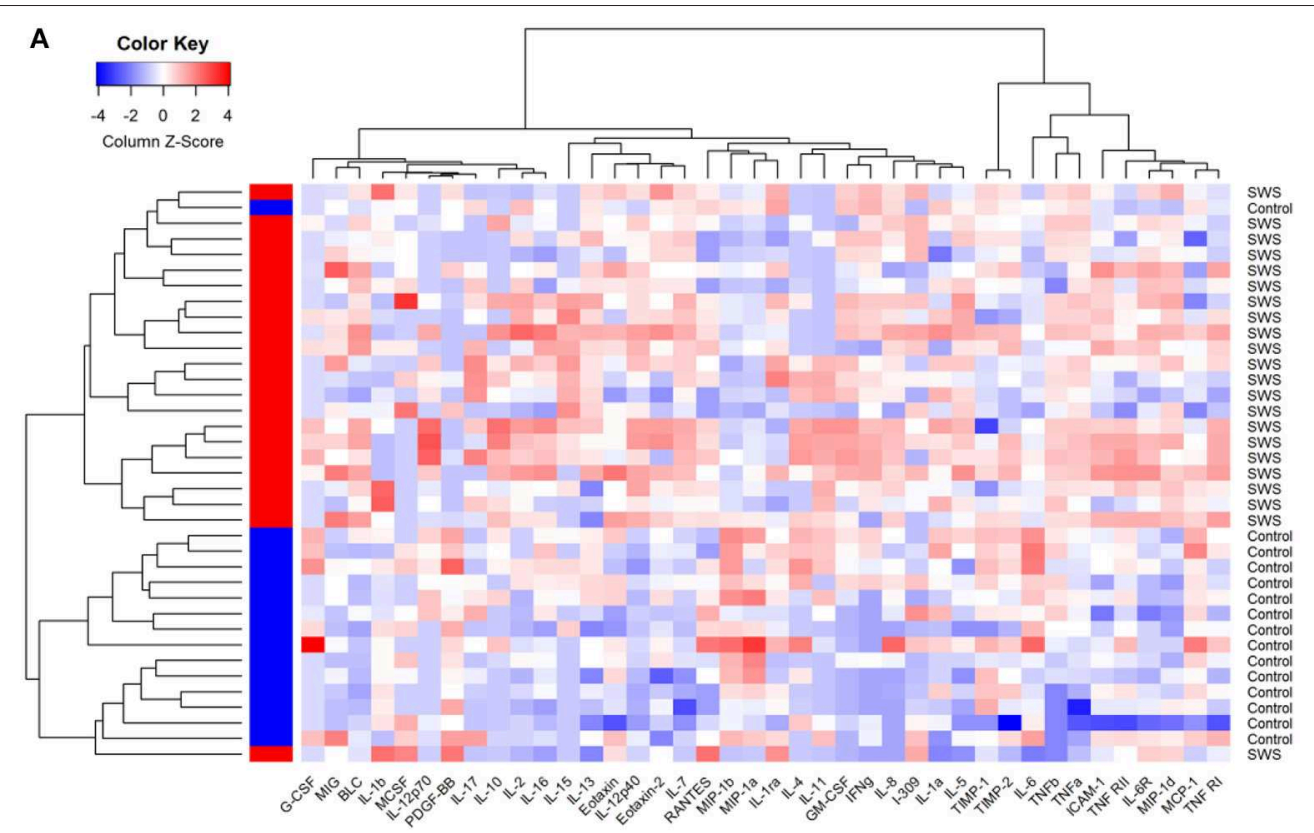

B

Principal Components Plot

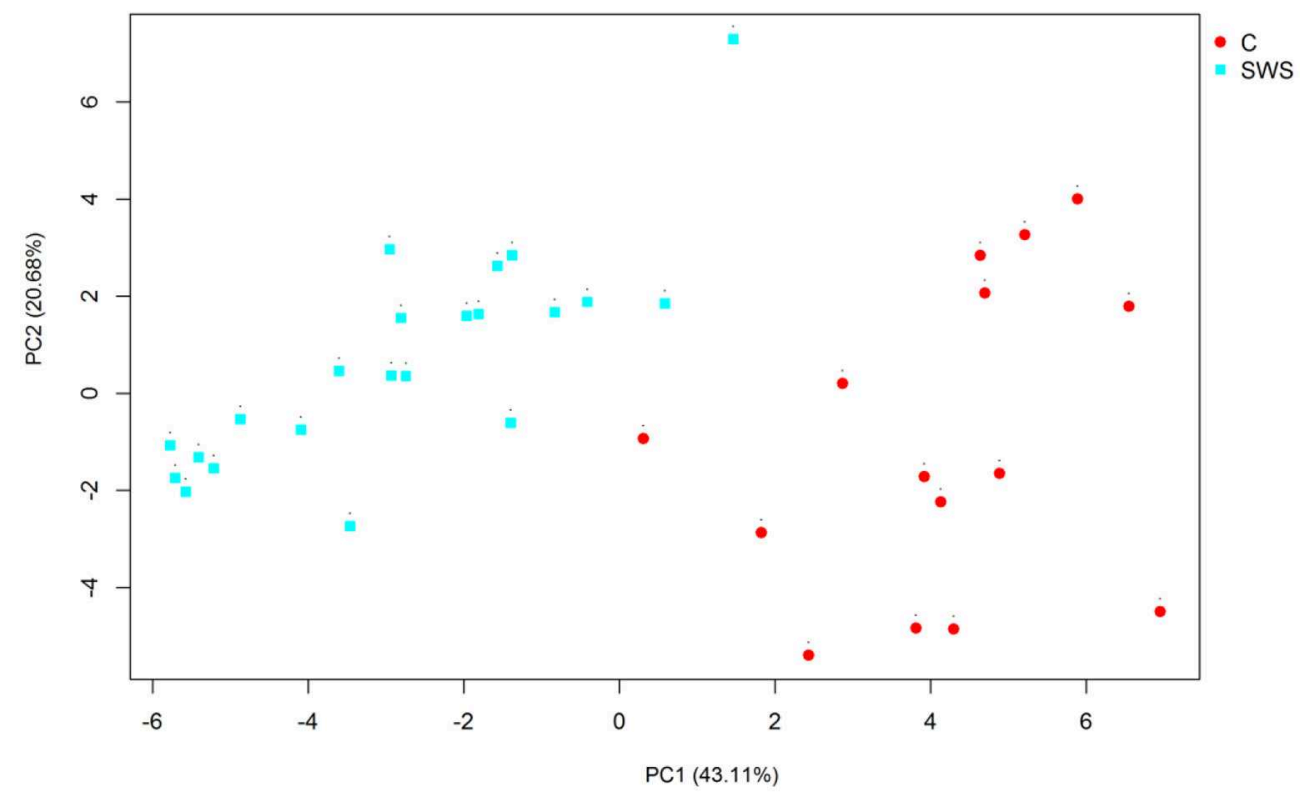

FIGURE 1 | (A) Hierarchical clustering heat map of cytokines in aqueous humor. Cytokine concentrations are depicted as colors ranging from blue to white to red, presenting low, intermediate, and high concentration, respectively, according to the mean of that cytokine. The group of the samples is showed by the label on the right and by the colored bar on the left (red represents SWS and blue represents control). (B) Principal component analysis of aqueous humor samples, with the axes representing two principal components (PC1 and PC2). The fraction of variation that is displayed by each axis is noted in its label. The SWS samples are sharply discriminated from the control samples on the second principal component (PC2) that accounts for $20.68 \%$ of all variability.

cytokines. The pathways enriched by the differential cytokines in aqueous humor samples are shown in Figure 4. The results demonstrated that the differential cytokines were significantly enriched in mainly four pathways including cytokine-cytokine receptor interaction $(P=1.33 \mathrm{E}-20)$, viral protein interaction with cytokine and cytokine receptor $(P=5.23 \mathrm{E}-14)$, JAK-STAT signaling pathway $(P=2.34 \mathrm{E}-10)$ and rheumatoid arthritis(RA) $(P=1.45 \mathrm{E}-10)$. 
TABLE 2 | Concentrations of cytokines in SG and control groups.

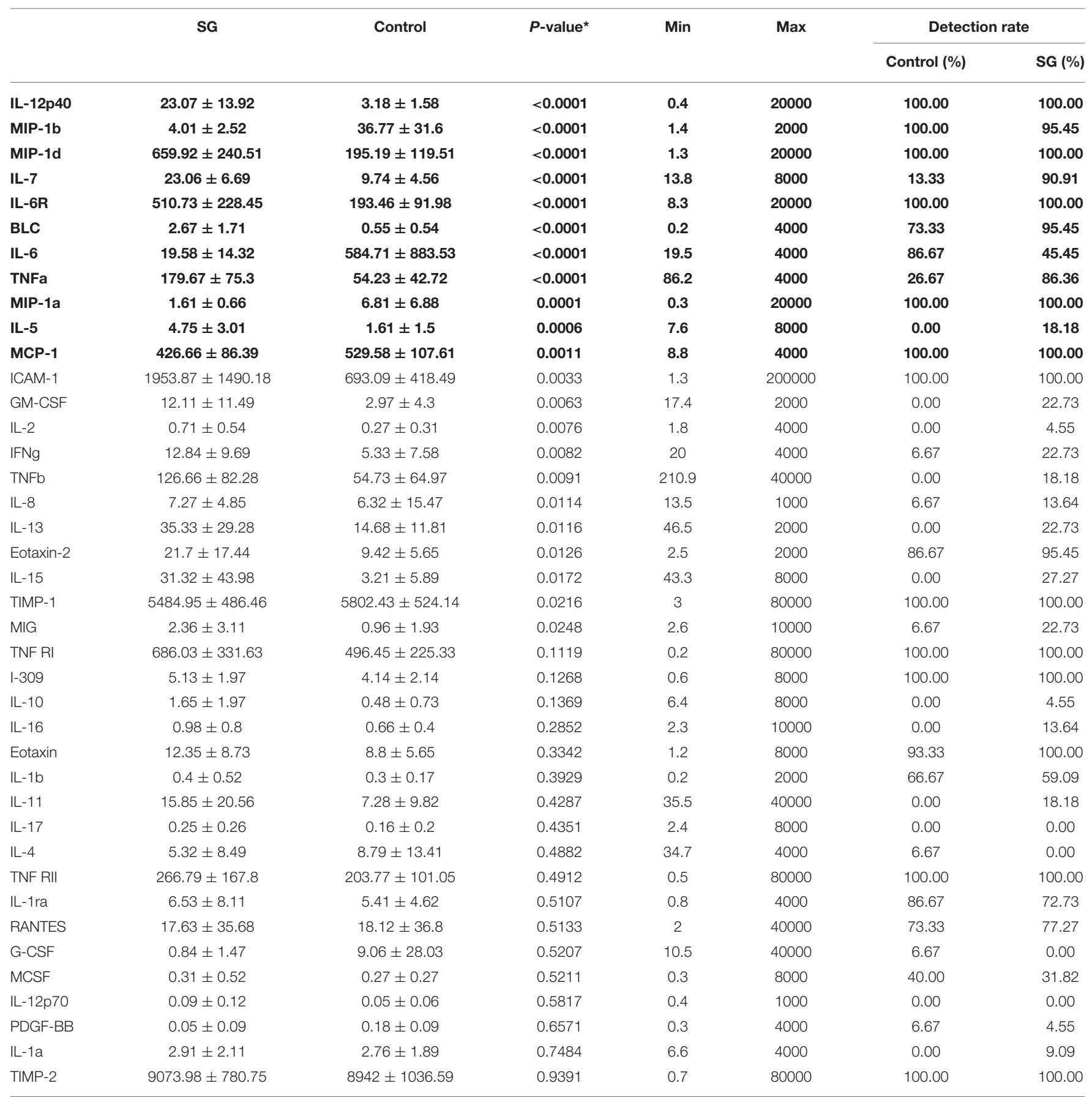

Control, senile cataract eyes; SG, Sturge-weber syndrome induced glaucoma;

Mean \pm SD; Cytokines: $(\mathrm{pg} / \mathrm{ml})$; ' Mann-Whitney U test;

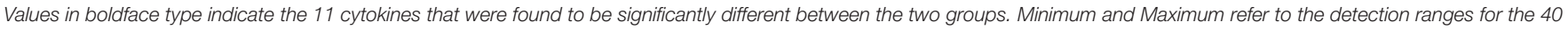
cytokines that were measured using the cytokine antibody arrays in accordance with the manufacturer's instructions. Detection rate shows the ratio of values within ranges to total.

\section{DISCUSSION}

In this study, we utilized the cytokine antibody array method to find out the cytokine profile of the AH in SWS-induced glaucoma (SG) and age-related cataract patients. We found that the cytokine profiles were significantly different between the two groups. The level of IL-12p40, MIP-1d, IL-7, IL-6R, BLC, TNF-a, IL-5 were elevated in the SG samples compared to the samples of the cataract patients. However, the concentration of MCP-1, IL-6, MIP-1b, MIP-1a were lower in the SG group. Furthermore, 

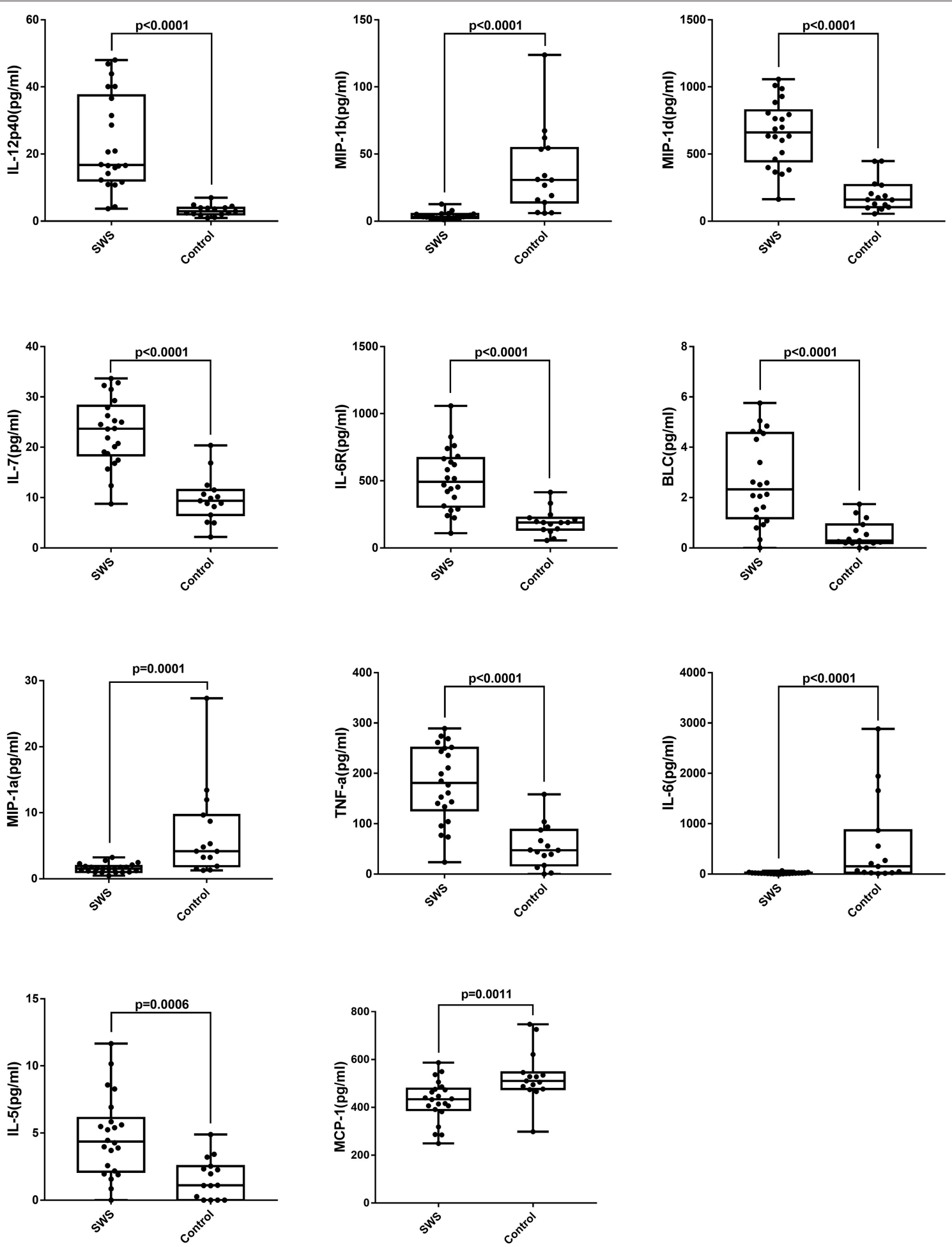

FIGURE 2 | Box plots of IL-5, IL-6, IL-7, IL-12p40, IL-6R, MIP-1a, MIP-1b, MIP-1d, BLC, MCP-1, TNF-a in patients with SWS and senile cataract. Dots represent individual values (Mann-Whitney $U$-test with Bonferroni correction for multiple comparisons).

we showed the cytokine level of four cytokines (IL-12p40, IL7, MIP-1a, and TNF-a) were correlated with clinical parameters (age and IOP) in the early-onset SG patients.
Previous studies revealed that significantly different aqueous humor inflammatory cytokine expression can appear in different types of glaucomatous eyes. In primary open angle glaucoma 
TABLE 3 | Correlation among 11 cytokines in SG patients.

\begin{tabular}{|c|c|c|c|c|c|c|c|c|c|c|c|}
\hline$\rho / P$-value & IL-12p40 & MIP-1b & MIP-1d & IL-7 & IL-6R & BLC & MIP-1a & IL-6 & TNF-a & IL-5 & MCP-1 \\
\hline IL-12p40 & & 0.2038 & 0.5325 & 0.5370 & 0.5743 & 0.8295 & 0.6951 & 0.5404 & 0.5449 & 0.3868 & 0.5460 \\
\hline MIP-1b & 0.3629 & & 0.1451 & 0.0762 & 0.3416 & 0.4602 & 0.6510 & 0.2016 & 0.0638 & -0.1101 & 0.2242 \\
\hline MIP-1d & 0.0107 & 0.5194 & & 0.2072 & 0.6115 & 0.4647 & 0.3936 & 0.0073 & 0.4195 & 0.2208 & 0.1158 \\
\hline IL-7 & 0.0100 & 0.7360 & 0.3548 & & 0.2513 & 0.3744 & 0.3665 & 0.5313 & 0.8069 & 0.6262 & 0.2772 \\
\hline IL-6R & 0.0052 & 0.1197 & 0.0025 & 0.2593 & & 0.7628 & 0.6849 & 0.1948 & 0.3732 & 0.1270 & 0.3642 \\
\hline BLC & $<0.0001$ & 0.0312 & 0.0293 & 0.0861 & $<0.0001$ & & 0.7775 & 0.3484 & 0.3416 & 0.2445 & 0.4082 \\
\hline MIP-1a & 0.0003 & 0.0010 & 0.0700 & 0.0935 & 0.0004 & $<0.0001$ & & 0.3190 & 0.3348 & 0.0604 & 0.5426 \\
\hline IL-6 & 0.0094 & 0.3683 & 0.9741 & 0.0109 & 0.3850 & 0.1121 & 0.1479 & & 0.5731 & 0.4816 & 0.6217 \\
\hline TNF-a & 0.0087 & 0.7779 & 0.0519 & $<0.0001$ & 0.0871 & 0.1197 & 0.1277 & 0.0053 & & 0.5776 & 0.3010 \\
\hline IL-5 & 0.0754 & 0.6257 & 0.3235 & 0.0018 & 0.5732 & 0.2728 & 0.7894 & 0.0232 & 0.0049 & & -0.0536 \\
\hline MCP-1 & 0.0086 & 0.3159 & 0.6080 & 0.2116 & 0.0956 & 0.0593 & 0.0091 & 0.0020 & 0.1735 & 0.8126 & \\
\hline
\end{tabular}

Correlation coefficient ( $\rho$ ) and P-values for each pair of cytokines are calculated by Spearman's correlation test.

$P<0.05$ was considered significant (bold).

TABLE 4 | Correlation of cytokines with age, IOP, C/D, or corneal diameter in early-onset SG group.

\begin{tabular}{|c|c|c|c|c|c|c|c|c|}
\hline & \multicolumn{2}{|c|}{ Age } & \multicolumn{2}{|c|}{ IOP } & \multicolumn{2}{|c|}{ C/D } & \multicolumn{2}{|c|}{ Corneal diameter } \\
\hline IL-12p40 & -0.7114 & 0.0028 & 0.3548 & 0.1776 & 0.1509 & 0.5770 & 0.2023 & 0.4492 \\
\hline MIP-1b & -0.1682 & 0.5336 & 0.3636 & 0.1662 & -0.1154 & 0.6704 & -0.4167 & 0.1090 \\
\hline MIP-1d & -0.3572 & 0.1744 & 0.4095 & 0.1153 & -0.2130 & 0.4283 & -0.0604 & 0.8240 \\
\hline BLC & -0.4554 & 0.0763 & 0.4080 & 0.1167 & 0.1021 & 0.7068 & 0.2461 & 0.3552 \\
\hline MIP-1a & -0.4866 & 0.0559 & 0.5277 & 0.0356 & 0.1923 & 0.4755 & -0.0619 & 0.8196 \\
\hline $\mathrm{IL}-6$ & -0.2962 & 0.2654 & 0.3577 & 0.1737 & -0.0118 & 0.9653 & 0.3564 & 0.1748 \\
\hline TNF-a & -0.2247 & 0.4027 & 0.5455 & 0.0289 & 0.2101 & 0.4349 & 0.0151 & 0.9573 \\
\hline IL-5 & -0.1369 & 0.6131 & 0.3237 & 0.2213 & 0.0399 & 0.8832 & 0.1842 & 0.4915 \\
\hline
\end{tabular}

IOP, intraocular pressure; $C / D$, cup-to-disc ratio;

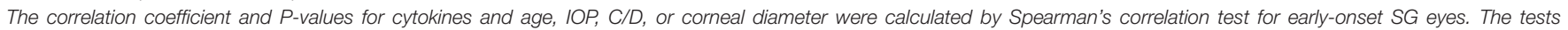
demonstrate correlation between cytokine levels (IL-12p40, IL-7, MIP-1a and TNF-a) and clinical parameters (age and IOP).

$P<0.05$ was considered significant (bold and italic).

(POAG) eyes, the level of IL-12, IL-6, TNF- $\alpha$, IL-8, IFN- $\gamma$ were noted to be remarkably raised (24-26). In pseudoexfoliation glaucoma (PEG) eyes, an increase of CCL13, CCL15, CCL22, CCL24, CXCL13, CXCL16, IL-4, IL-6, IL-8, and IL-16 were observed $(27,28)$. For uveitic glaucomatous eyes, IL-6, IL-8, VEGF, CCL2, and TNF- $\alpha$ were detected to be higher compared to the controls (29). Also, in the neovascular glaucoma (NVG) patients, increased level of IL-6, IL-8, PDGF, CCL2, and TNF$\alpha$ in $\mathrm{AH}$ were previously reported (30). In our study, there was a significant difference in the inflammation-related cytokine expression between the SG and cataract patients, presenting a specific cytokine profile in the aqueous of SWS patients, indicating that cytokine-mediated inflammation was involved in the development of SG.

IL-12 is a proinflammatory cytokine which promote Th1 cell differentiation and have a pivotal role in $\mathrm{T}$ cell-mediated response. It is composed of two subunits (IL12-p40 and IL12p35), and is mainly secreted from activated antigen-presenting cells (APCs), for example, dendritic cells (DCs) (31). The level of IL-12p40 has been reported to be increased in many conditions of autoimmune disorders, such as psoriasis, rheumatoid arthritis (RA), Crohn's disease (32) and uveitis (33). In addition, inflammatory and angiogenic processes are recognized as the crucial factors to the development of diabetic retinopathy (DR) and diabetic macular edema (DME). Moreover, patients with DR or DME have a higher concentration of IL-12 in the AH when compared with controls (34). Consistently, it has been previously shown that DME patients have lower levels of IL12 after treatment (35). In the current study, the level of IL$12 \mathrm{p} 40$ in the $\mathrm{AH}$ of SG patients were notably higher compared with controls, indicating that the inflammatory and angiogenic function of IL-12 may contribute to pathogenesis of SG. 

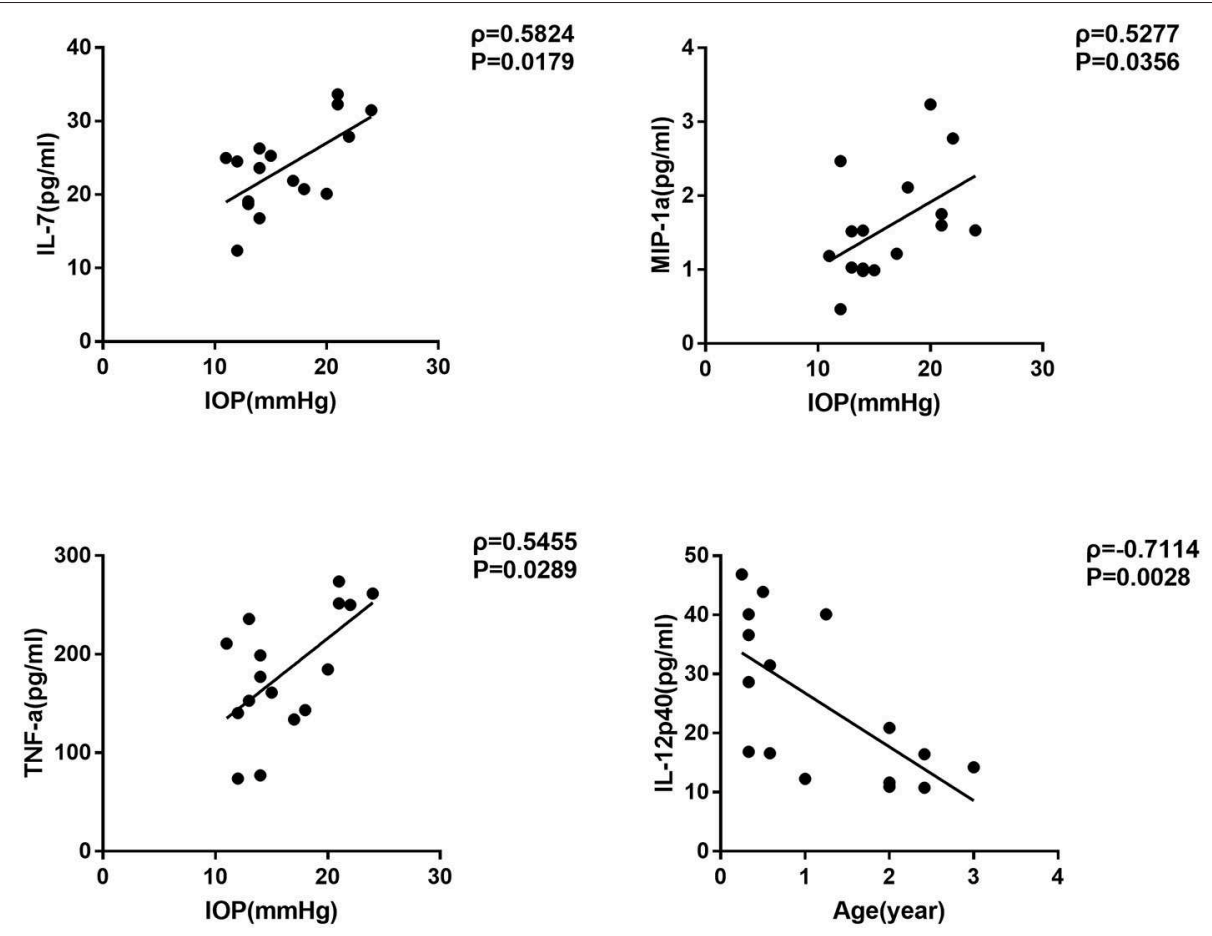

FIGURE 3 | Scatterplots showing the correlation of four selected cytokines with IOP and age in early-onset SG subgroup. Correlations between IOP and, respectively: IL-7 $(\rho=0.5824, P=0.0179)$, MIP-1a $(\rho=0.5277, P=0.0356)$, TNF-a $(\rho=0.5455, P=0.0289)$. Additionally, Correlations between age and IL-12p40 ( $\rho=$ $-0.7114, P=0.0028)$ (Spearman's correlation test). The solid line represents the linear regression line.

MIP-1d(CCL15) is secreted by various cells (36) in normal condition and is elevated in autoimmune disorders such as RA (37) and asthma (38). MIP-1d can stimulate the migration and differentiation of the chemotactic endothelial cell, and it has a pivotal role in angiogenesis (39). Moreover, MIP-1d was reported to be important in tumor progression by recruiting suppressive monocytes to facilitate immune escape and angiogenesis (40). Our results showed that MIP-1d level was significantly higher in SWS group. Considering the important role of vascular malformation in the facial, leptomeningeal, choroidal lesions of SWS, we hypothesize that ocular (scleral and/or choroidal) angiogenesis may contribute to the pathogenesis in ocular hypertension and RGC degeneration of SG. If proved in future animal experiments or clinical trials, MIP-1d could be of use to the treatment of SWS-induce choroidal hemangioma and glaucoma.

MCP-1(CCL2), MIP-1 $\alpha$ (CCL3), and MIP-1 $\beta$ (CCL4) act as typical chemoattractant that is involved in the inflammatory reaction by recruiting different immune cells to the injured area $(41,42)$. Additionally, they were previously reported to be increased in many pathological conditions of ocular inflammation, including glaucoma (43) and polypoidal choroidal vasculopathy (PCV) (44). Interestingly, in the present study, we found decreased MCP-1, MIP- $1 \alpha$, and MIP- $1 \beta$ concentrations in the eyes of SG patients when compared with the cataract eyes, indicating a conceivable 'atypical inflammation' in the aqueous humor during the disease progression. The explanation for the discrepancy may be as follows: on the one hand, previous studies showed MCP-1 was notably elevated in $\mathrm{AH}$ in the contralateral eye following the first-eye cataract surgery (45). In our study, among the 15 cataract patients, 9 patients had already had phacoemulsification for the contralateral eyes before the aqueous samples were taken, which may induce the relatively higher concentration of chemokines detected in the cataract group. On the other hand, the normal trabecular meshwork endothelial cells constitutively secrete chemokines, which maintain homeostasis of the outflow of the aqueous humor (46). Hence, the constitutive secretion of these chemokines may be affected by the damaged trabecular meshwork endothelial cells in the SWS-induced glaucomatous progression.

Tumor necrosis factor-alpha (TNF-a) is a proinflammatory cytokine with pleiotropic effects on various cell types. Enhanced TNF-a expression have been previously reported in $\mathrm{AH}$, trabecular meshwork, retina and optic nerve of clinical glaucomatous specimen, which is consistent with our present finding $(19,25,47-49)$. IL-6 is also a proinflammatory cytokine that promotes inflammatory responses and is involved in woundhealing and leucocyte recruitment $(50,51)$. Many studies of different types glaucoma have shown an increased level of IL-6 in aqueous humor $(24,29,30)$. However, in the current study, our result showed a decreased level of IL- 6 in SWS patients. An explanation of the inconsistency might be that cytokines are expressed in a complicated regulatory pattern, which can be affected by a variety of microenvironmental stimuli, and 


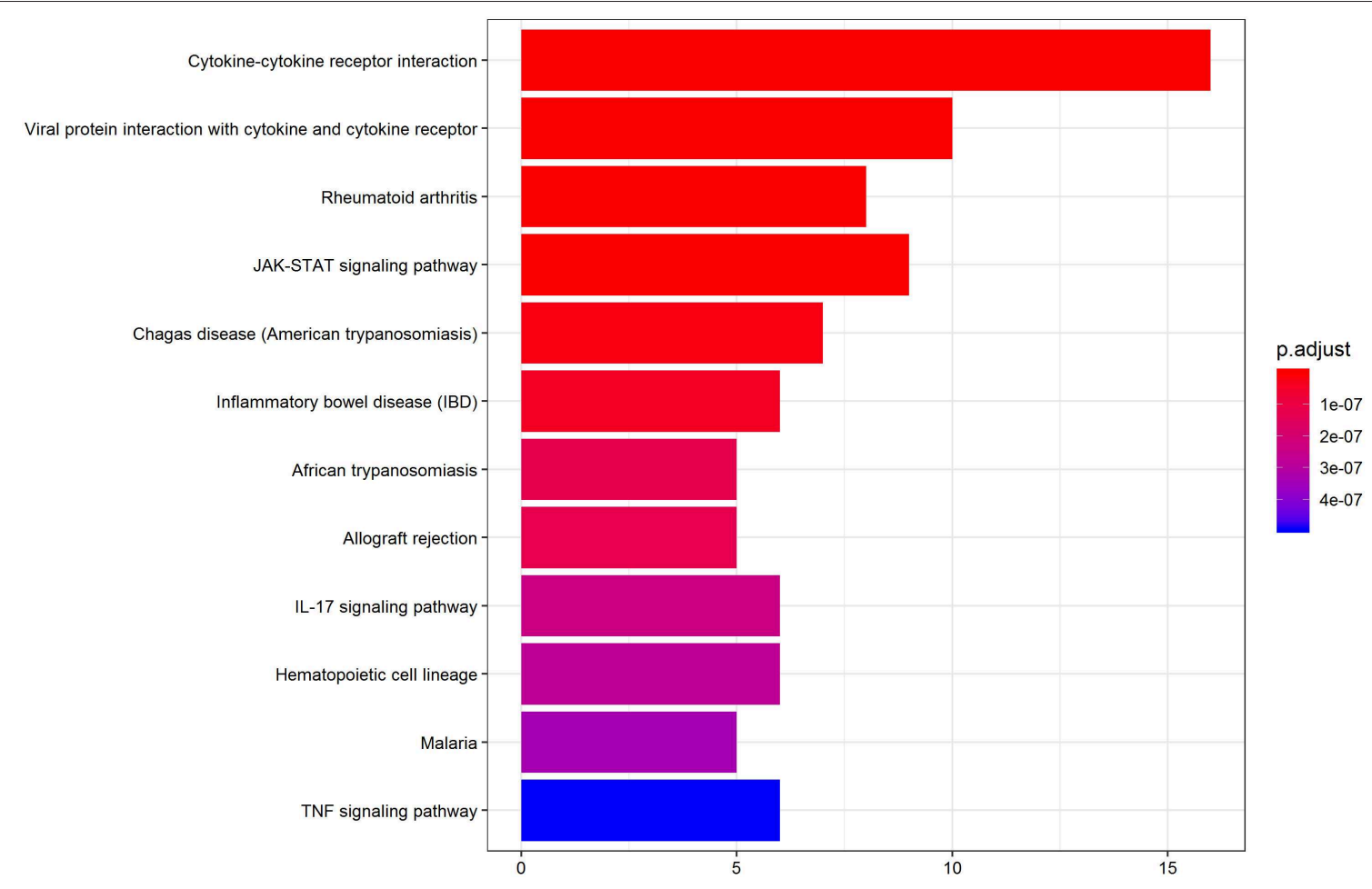

FIGURE 4 | KEGG pathway analysis indicates the significant pathways enriched by differentially expressed cytokines in AH of patients. Top 12 pathways for the differentially expressed cytokines are displayed. The $\mathrm{x}$-axis shows the amounts of cytokines associated with KEGG pathway. The color ranging from blue to red presents different level of adjusted $p$-value.

might therefore differ quite considerably in another disease subtype or in another course of disease. It is noteworthy that, in our study, we observed significantly higher levels of IL-6R in the AH of SG patients. It has been shown that soluble IL6ra (sIL-6Ra) can interact with IL-6, further constituting the IL-6/sIL-6Ra complex, which can bind to glycoprotein-130 on cells and then induce IL-6 trans-signaling (52). Additionally, IL6 trans-signaling can increase IL- 6 activity under inflammatory environment and to further prevent the apoptosis of $\mathrm{T}$ cells, which probably play a crucial role in the exacerbation and the prolongation of autoimmune disease processes (5355). Currently, biologic agents of anti-TNF- $\alpha$ and anti-IL6 receptor antibodies are widely used for uveitis treatment with satisfying clinical outcomes (56-58). Therefore, there is a possibly important role for these cytokine-related inflammations to play in the pathogenesis of SG, which could provide the therapeutically potential target for SG in the near future.

Previous studies reported significant correlations between cytokines and clinical parameters of glaucoma. In POAG and PEG patients, TGF- $\beta$, IL- 8 , and SAA were positively correlated with pre-operative IOP, and IL-6 was positively correlated with age (59) There was also a report that found IL-8, IP-10, CCL2, and CCL4 were correlated with IOP in POAG and NVG eyes (43). Moreover, the positive correlation of G-CSF level between IOP has been reported in $\mathrm{AH}$ of acute primary angle-closure (APAC) patients (60). In the current study, initially, there were no cytokines correlated with IOP in SG patients. Considering that bimodal-onset glaucoma may be involved in different pathogenesis, we further classified the SWS group into the earlyonset (age of surgery $<4$ years) and late-onset (age of surgery $>4$ years) groups. Surprisingly, we noted that the level of IL-7, MIP1a, and TNF-a were positively correlated with the preoperative IOP in eyes of early-onset SG. Coincidently, a recent study of rheumatoid arthritis (RA) showed that the high responsiveness to IL-7 stimulation together with the synergy of IL-7 and M1-promoting factors (e.g., MIP-1b, TNF-a) can enhance the expression of joint myeloid IL-7R and further exacerbate arthritis (61). Consistently, our KEGG analysis also suggested that RA and SG may have the same cytokine microenvironment in common (Figure 4). Additionally, we found IL12-p40 was significantly negatively correlated with age in SWS group, which implicated that the IL-12 mediated-inflammation may be associated with the development of early-onset SG. Taken together, the result indicated that the intricate cytokine networks may play a pivotal role in pathogenesis and progression of early-onset SG, although the specific mechanism of interactions among these cytokines needed to be further validated.

The current research did have several limiting factors. First, a relatively small sample size may lead to statistical bias. However, the small sample size may result from the strict selection criteria ensuring the same immunological background of our subjects, as well as the rarity of disease. Second, the age difference 
between two groups was large, which was partially responsible for the differences of cytokine profile. Even though we were fully aware that age-matched non-glaucoma infant with SWS may be the ideal choice for control group, they were not available in the current study for the ethical issues. Nevertheless, after statistical adjustment, we could at least draw a tentative conclusion that altered cytokine levels is mainly caused by the pathology of disease rather than the age-associated bias. Third, no functional analysis conducted to elucidate the exact mechanism between these altered cytokines and IOP elevation. Since the very small volume of samples and unidentified cell type or animal model in SG pathogenesis, we can hardly further investigate the immunopathogenesis of altered cytokine profile in SG. Further studies utilizing intraocular tissue specimen of SG patients or developed animal models may be conducive to exploring the potential roles of cytokine-related inflammation and angiogenesis in the pathological process of SG.

In conclusion, the present study firstly showed the characteristic cytokine profiles of aqueous humor in patients with SG. Additionally, the intraocular levels of a wide variety of cytokines were related to clinical parameters, such as age and IOP. Further investigations including animal experiments and clinical trials are necessary to explore the relative importance and mutual mechanism of these cytokines.

\section{DATA AVAILABILITY STATEMENT}

The datasets generated for this study are available on request to the corresponding author.

\section{REFERENCES}

1. Comi A. Current therapeutic options in sturge-weber Syndrome. Semin Pediatr Neurol. (2015) 22:295-301. doi: 10.1016/j.spen.2015.10.005

2. Shirley MD, Tang H, Gallione CJ, Baugher JD, Frelin LP, Cohen B, et al. Sturge-Weber syndrome and port-wine stains caused by somatic mutation in GNAQ. N Engl J Med. (2013) 368:1971-9. doi: 10.1056/NEJMoa12 13507

3. Poduri, Evrony GD, Cai X, Walsh CA. Somatic mutation, genomic variation, and neurological disease. Science. (2013) 341:1237758. doi: $10.1126 /$ science. 1237758

4. Groh J, Martini R. Neuroinflammation as modifier of genetically caused neurological disorders of the central nervous system: understanding pathogenesis and chances for treatment. Glia. (2017) 65:1407-22. doi: $10.1002 /$ glia. 23162

5. Klein RS, Garber C, Howard N. Infectious immunity in the central nervous system and brain function. Nat Immunol. (2017) 18:132-41. doi: 10.1038/ni.3656

6. Bosemani T, Huisman TA, Poretti A. pediatric neurocutaneous syndromes with cerebellar involvement. Neuroimaging Clin North Am. (2016) 26:417-34. doi: 10.1016/j.nic.2016.03.008

7. Park SJ, Sawitzki B, Kluwe L, Mautner VF, Holtkamp N, Kurtz A. Serum biomarkers for neurofibromatosis type 1 and early detection of malignant peripheral nerve-sheath tumors. BMC Med. (2013) 11:109. doi: 10.1186/1741-7015-11-109

8. Maldonado M, Baybis M, Newman D, Kolson DL, Chen W, McKhann G II, et al. Expression of ICAM-1, TNF-alpha, NF kappa B, and MAP kinase in tubers of the tuberous sclerosis complex. Neurobiol Dis. (2003) 14:279-90. doi: 10.1016/S0969-9961(03)00127-X

\section{ETHICS STATEMENT}

The studies involving human participants were reviewed and approved by Institutional Review Board of Ninth People's Hospital, Shanghai Jiao Tong University School of Medicine. Written informed consent to participate in this study was provided by the participants' legal guardian/next of kin.

\section{AUTHOR CONTRIBUTIONS}

$\mathrm{CP}$ and WG designed the study. WG, CP, LX, XD, and CZ collected aqueous humor samples. CP, YW, and DC performed the measurements and data analysis. CP, YW, and WG wrote the manuscript. All authors have read and critically revised the manuscript.

\section{FUNDING}

This work was supported by the National Natural Science Foundation (81670845, 81970796), National Key R\&D Program of China (2018YFC1106100, 2018YFC1106101), and the Research Grant of the Shanghai Science and Technology Committee (17DZ2260100).

\section{SUPPLEMENTARY MATERIAL}

The Supplementary Material for this article can be found online at: https://www.frontiersin.org/articles/10.3389/fimmu. 2020.00004/full\#supplementary-material

9. Boer K, Crino PB, Gorter JA, Nellist M, Jansen FE, Spliet WG, et al. Gene expression analysis of tuberous sclerosis complex cortical tubers reveals increased expression of adhesion and inflammatory factors. Brain Pathol. (2010) 20:704-19. doi: 10.1111/j.1750-3639.2009.00341.x

10. Sullivan TJ, Clarke MP, Morin JD. The ocular manifestations of the SturgeWeber syndrome. J Pediatr Ophthalmol Strab. (1992) 29:349-56.

11. Sujansky E, Conradi S. Sturge-Weber syndrome: age of onset of seizures and glaucoma and the prognosis for affected children. J Child Neurol. (1995) 10:49-58. doi: 10.1177/088307389501000113

12. Phelps CD. The pathogenesis of glaucoma in Sturge-Weber syndrome. Ophthalmology. (1978) 85:276-86. doi: 10.1016/S0161-6420(78)35667-0

13. Cibis GW, Tripathi RC, Tripathi BJ. Glaucoma in Sturge-Weber syndrome. Ophthalmology. (1984) 91:1061-71. doi: 10.1016/S0161-6420(84)34194-X

14. Wu Y, Yu RJ, Chen D, Xu L, Li M, Zhu L, et al. Glaucoma in patients with eyes close to areas affected by port-wine stain has lateral and gender predilection. Chin Med J. (2017) 130:2922-6. doi: 10.4103/0366-6999.220319

15. Stamer WD, Acott TS. Current understanding of conventional outflow dysfunction in glaucoma. Curr Opin Ophthalmol. (2012) 23:135-43. doi: 10.1097/ICU.0b013e32834ff23e

16. Alvarado JA, Murphy CG. Outflow obstruction in pigmentary and primary open angle glaucoma. Arch Ophthalmol. (1992) 110:1769-78. doi: 10.1001/archopht.1992.01080240109042

17. Lutjen-Drecoll E, Shimizu T, Rohrbach M, Rohen JW. Quantitative analysis of 'plaque material' in the inner- and outer wall of Schlemm's canal in normal- and glaucomatous eyes. Exp Eye Res. (1986) 42:443-55. doi: 10.1016/0014-4835(86)90004-7

18. Cvenkel, Zupan M, Hvala A. Transmission electron microscopic analysis of trabecular meshwork in secondary glaucoma after intravitreal silicone oil injection. Int Ophthalmol. (1996) 20:43-7. doi: 10.1007/BF00212944 
19. Taurone S, Ripandelli G, Pacella E, Bianchi E, Plateroti AM, De Vito S, et al. Potential regulatory molecules in the human trabecular meshwork of patients with glaucoma: immunohistochemical profile of a number of inflammatory cytokines. Mol Med Rep. (2015) 11:1384-90. doi: 10.3892/mmr.2014.2772

20. Choi JA, Kim JE, Noh SJ, Kyoung Kim E, Park CK, et al. Enhanced cytomegalovirus infection in human trabecular meshwork cells and its implication in glaucoma pathogenesis. Sci Rep. (2017) 7:43349. doi: 10.1038/srep43349

21. Liton PB, Luna C, Bodman M, Hong A, Epstein DL, Gonzalez P. Induction of IL-6 expression by mechanical stress in the trabecular meshwork. Biochem Biophys Res Commun. (2005) 337:1229-36. doi: 10.1016/j.bbrc.2005.09.182

22. Chen H, Cho KS, Vu HK, Shen CH, Kaur M, Chen G, et al. Commensal microflora-induced $\mathrm{T}$ cell responses mediate progressive neurodegeneration in glaucoma. Nat Commun. (2018) 9:3209. doi: 10.1038/s41467-018-06428-2

23. Wu Y, Yu R, Chen D, Xu L, Zhu L, Li M, et al. Early trabeculotomy ab externo in treatment of sturge-weber Syndrome. Am J Ophthalmol. (2017) 182:141-6. doi: 10.1016/j.ajo.2017.08.002

24. Chua J, Vania M, Cheung CM, Ang M, Chee SP, Yang H, et al. Expression profile of inflammatory cytokines in aqueous from glaucomatous eyes. $\mathrm{Mol}$ Vision. (2012) 18:431-8.

25. Sawada H, Fukuchi T, Tanaka T, Abe H. Tumor necrosis factor-alpha concentrations in the aqueous humor of patients with glaucoma. Investig Ophthalmol Vis Sci. (2010) 51:903-6. doi: 10.1167/iovs.09-4247

26. Kuchtey J, Rezaei KA, Jaru-Ampornpan P, Sternberg P, Kuchtey RW. Multiplex cytokine analysis reveals elevated concentration of interleukin-8 in glaucomatous aqueous humor. Investig Ophthalmol Vis Sci. (2010) 51:6441-7. doi: 10.1167 /iovs.10-5216

27. Garweg JG, Zandi S, Pfister IB, Skowronska M, Gerhardt C. Comparison of cytokine profiles in the aqueous humor of eyes with pseudoexfoliation syndrome and glaucoma. PLOS ONE. (2017) 12:e0182571. doi: 10.1371/journal.pone.0182571

28. Zenkel M, Lewczuk P, Jünemann A, Kruse FE, Naumann GO, SchlötzerSchrehardt U. Proinflammatory cytokines are involved in the initiation of the abnormal matrix process in pseudoexfoliation syndrome/glaucoma. Am J Pathol. (2010) 176:2868-79. doi: 10.2353/ajpath.2010.090914

29. Ohira S, Inoue $\mathrm{T}$, Iwao $\mathrm{K}$, Takahashi E, Tanihara H. Factors Influencing Aqueous Proinflammatory Cytokines and Growth Factors in Uveitic Glaucoma. PLoS ONE. (2016) 11:e0147080. doi: 10.1371/journal.pone. 0147080

30. Ohira $\mathrm{S}$, Inoue $\mathrm{T}$, Shobayashi $\mathrm{K}$, Iwao $\mathrm{K}$, Fukushima $\mathrm{M}$, Tanihara H. Simultaneous increase in multiple proinflammatory cytokines in the aqueous humor in neovascular glaucoma with and without intravitreal bevacizumab injection. Investig Ophthalmol Vis Sci. (2015) 56:3541-8. doi: 10.1167/iovs.14-15918

31. Oshikiri Y, Nara H, Takeda Y, Araki A, Nemoto N, Gazi MY, et al. Interleukin12 p40 variant form reduces Interleukin-12p80 secretion. Cytokine. (2019) 120:251-7. doi: 10.1016/j.cyto.2019.05.017

32. Teng MW, Bowman EP, McElwee JJ, Smyth MJ, Casanova JL, Cooper AM, et al. IL-12 and IL-23 cytokines: from discovery to targeted therapies for immune-mediated inflammatory diseases. Nat Med. (2015) 21:719-29. doi: $10.1038 / \mathrm{nm} .3895$

33. Abu El-Asrar AM, Berghmans N, Al-Obeidan SA, Gikandi PW, Opdenakker G, Van Damme J, et al. Local cytokine expression profiling in patients with specific autoimmune uveitic entities. Ocular Immunol Inflamm. (2019). doi: 10.1080/09273948.2019.1604974. [Epub ahead of print].

34. Gverovic Antunica, Karaman K, Znaor L, Sapunar A, Busko V, Puzovic V. IL-12 concentrations in the aqueous humor and serum of diabetic retinopathy patients. Graefe's Arch Clin Exp Ophthalmol. (2012) 250:815-21. doi: 10.1007/s00417-011-1905-4

35. Lim SW, Bandala-Sanchez E, Kolic M, Rogers SL, McAuley AK, Lim LL, et al. The influence of intravitreal ranibizumab on inflammationassociated cytokine concentrations in eyes with diabetic macular edema. Invest Ophthalmol Vis Sci. (2018) 59:5382-90. doi: 10.1167/iovs.17-23325

36. Coulin F, Power CA, Alouani S, Peitsch MC, Schroeder JM, Moshizuki $\mathrm{M}$, et al. Characterisation of macrophage inflammatory protein5/human CC cytokine-2, a member of the macrophage-inflammatoryprotein family of chemokines. Eur J Biochem. (1997) 248:507-15. doi: 10.1111/j.1432-1033.1997.00507.x
37. Berahovich RD, Miao Z, Wang Y, Premack B, Howard MC, Schall TJ. Proteolytic activation of alternative CCR1 ligands in inflammation. $J$ Immunol. (2005) 174:7341-51. doi: 10.4049/jimmunol.174.11.7341

38. Shimizu Y, Dobashi K. CC-chemokine CCL15 expression and possible implications for the pathogenesis of IgE-related severe asthma. Mediators Inflamm. (2012) 2012:475253. doi: 10.1155/2012/475253

39. Hwang J, Kim CW, Son KN, Han KY, Lee KH, Kleinman HK, et al. Angiogenic activity of human CC chemokine CCL15 in vitro and in vivo. FEBS Lett. (2004) 570:47-51. doi: 10.1016/j.febslet.2004.06.023

40. Liu LZ, Zhang Z, Zheng BH, Shi Y, Duan M, Ma LJ, et al. CCL15 recruits suppressive monocytes to facilitate immune escape and disease progression in hepatocellular carcinoma. Hepatology. (2019) 69:143-59. doi: 10.1002/hep.30134

41. Bystry RS, Aluvihare V, Welch KA, Kallikourdis M, Betz AG. B cells and professional APCs recruit regulatory T cells via CCL4. Nat Immunol. (2001) 2:1126-32. doi: 10.1038/ni735

42. Maurer $\mathrm{M}$, von Stebut E. Macrophage inflammatory protein-1. Int J Biochem Cell Biol. (2004) 36:1882-6. doi: 10.1016/j.biocel.2003. 10.019

43. Kokubun T, Tsuda S, Kunikata H, Yasuda M, Himori N, Kunimatsu-Sanuki $S$, et al. Characteristic profiles of inflammatory cytokines in the aqueous humor of glaucomatous eyes. Ocular Immunol Inflamm. (2018) 26:1177-1188. doi: 10.1080/09273948.2017.1327605

44. Agrawal R, Balne PK, Wei X, Bijin VA, Lee B, Ghosh A, et al. Cytokine profiling in patients with exudative age-related macular degeneration and polypoidal choroidal vasculopathy. Invest Ophthalmol Vis Sci. (2019) 60:37682. doi: $10.1167 /$ iovs.18-24387

45. Zhu XJ, Wolff D, Zhang KK, He WW, Sun XH, Lu Y, et al. Molecular inflammation in the contralateral eye after cataract surgery in the first eye. Invest Ophthalmol Vis Sci. (2015) 56:5566-73. doi: 10.1167/iovs.1516531

46. Shifera AS, Trivedi S, Chau P, Bonnemaison LH, Iguchi R, Alvarado JA. Constitutive secretion of chemokines by cultured human trabecular meshwork cells. Exp Eye Res. (2010) 91:42-7. doi: 10.1016/j.exer.2010. 04.001

47. Yuan L, Neufeld AH. Tumor necrosis factor-alpha: a potentially neurodestructive cytokine produced by glia in the human glaucomatous optic nerve head. Glia. (2000) 32:42-50. doi: 10.1002/1098-1136(200010)32:1<42::AID-GLIA40>3.0.CO;2-3

48. Tezel G, Li LY, Patil RV, Wax MB. TNF-alpha and TNF-alpha receptor-1 in the retina of normal and glaucomatous eyes. Invest Ophthalmol Vis Sci. (2001) 42:1787-94.

49. Balaiya S, Edwards J, Tillis T, Khetpal V, Chalam KV. Tumor necrosis factor-alpha (TNF-alpha) levels in aqueous humor of primary open angle glaucoma. Clin Ophthalmol. (2011) 5:553-6. doi: 10.2147/OPTH. S19453

50. Romano M, Sironi M, Toniatti C, Polentarutti N, Fruscella P, Ghezzi P, et al. Role of IL-6 and its soluble receptor in induction of chemokines and leukocyte recruitment. Immunity. (1997) 6:315-25. doi: 10.1016/S1074-7613(00)80334-9

51. Wu WC, Hu DN, Gao HX, Chen M, Wang D, Rosen R, et al. Subtoxic levels hydrogen peroxide-induced production of interleukin- 6 by retinal pigment epithelial cells. Mol Vis. (2010) 16:1864-73.

52. Rose-John S. IL-6 trans-signaling via the soluble IL-6 receptor: importance for the pro-inflammatory activities of IL-6. Int J Biol Sci. (2012) 8:1237-47. doi: $10.7150 /$ ijbs.4989

53. Nowell MA, Richards PJ, Horiuchi S, Yamamoto N, Rose-John S, Topley N, et al. Soluble IL-6 receptor governs IL-6 activity in experimental arthritis: blockade of arthritis severity by soluble glycoprotein 130. J Immunol. (2003) 171:3202-9. doi: 10.4049/jimmunol.171.6.3202

54. Curnow SJ, Scheel-Toellner D, Jenkinson W, Raza K, Durrani OM, Faint $\mathrm{JM}$, et al. Inhibition of $\mathrm{T}$ cell apoptosis in the aqueous humor of patients with uveitis by IL-6/soluble IL-6 receptor trans-signaling. J Immunol. (2004) 173:5290-7. doi: 10.4049/jimmunol.173.8.5290

55. Barkhausen T, Tschernig T, Rosenstiel P, van Griensven M, Vonberg RP, Dorsch M, et al. Selective blockade of interleukin- 6 trans-signaling improves survival in a murine polymicrobial sepsis model. Critic Care Med. (2011) 39:1407-13. doi: 10.1097/CCM.0b013e318211ff56 
56. Levy-Clarke G, Jabs DA, Read RW, Rosenbaum JT, Vitale A, Van Gelder RN. Expert panel recommendations for the use of anti-tumor necrosis factor biologic agents in patients with ocular inflammatory disorders. Ophthalmology. (2014) 121:785-96 e3. doi: 10.1016/j.ophtha.2013. 09.048

57. Al Rashidi S, Al Fawaz A, Kangave D, Abu El-Asrar AM. Long-term clinical outcomes in patients with refractory uveitis associated with Behcet disease treated with infliximab. Ocular Immunol Inflamm. (2013) 21:468-74. doi: 10.3109/09273948.2013.779727

58. Mesquida M, Molins B, Llorenç V, de la Maza MS, Adán A. Targeting interleukin-6 in autoimmune uveitis. Autoimmun Rev. (2017) 16:1079-89. doi: 10.1016/j.autrev.2017.08.002

59. Takai Y, Tanito M, Ohira A. Multiplex cytokine analysis of aqueous humor in eyes with primary open-angle glaucoma, exfoliation glaucoma, and cataract. Investig Ophthalmol Vis Sci. (2012) 53:241-7. doi: 10.1167/iovs.1 $1-8434$

60. Du S, Huang W, Zhang X, Wang J, Wang W, Lam DSC. Multiplex cytokine levels of aqueous humor in acute primary angle-closure patients: fellow eye comparison. BMC Ophthalmol. (2016) 16:6. doi: 10.1186/s12886-0160182-8

61. Kim SJ, Chang HJ, Volin MV, Umar S, Van Raemdonck K, Chevalier A, et al. Macrophages are the primary effector cells in IL-7-induced arthritis. Cell Mol Immunol. (2019). doi: 10.1038/s41423-019-0235-z. [Epub ahead of print].

Conflict of Interest: The authors declare that the research was conducted in the absence of any commercial or financial relationships that could be construed as a potential conflict of interest.

Copyright (c) 2020 Peng, Wu, Ding, Chen, Zeng, Xu and Guo. This is an open-access article distributed under the terms of the Creative Commons Attribution License (CC $B Y)$. The use, distribution or reproduction in other forums is permitted, provided the original author(s) and the copyright owner(s) are credited and that the original publication in this journal is cited, in accordance with accepted academic practice. No use, distribution or reproduction is permitted which does not comply with these terms. 